\title{
Revisiting Strategies for Maternal Health Care in the Face of COVID-19 Pandemic Shrestha $N,{ }^{1}$ Dangal $\mathrm{G}^{2}$
}

\author{
${ }^{1}$ Department of Community Medicine, \\ Chitwan Medical College, \\ Bharatpur, Nepal. \\ ${ }^{2}$ Department of Obstetrics and Gynaecology, \\ Kathmandu Model Hospital, \\ Kathmandu, Nepal.
}

\section{Corresponding Author}

Niki Shrestha

Department of Community Medicine,

Chitwan Medical College,

Bharatpur, Nepal.

E-mail: drnikis1@gmail.com

\section{Citation}

Shrestha N, Dangal G. Revisiting Strategies for Maternal Health Care in the Face of COVID-19 Pandemic. Kathmandu Univ Med J. 2020;COVID-19 Special Issue 70(2):62-7.

\begin{abstract}
Novel coronavirus (SARS-CoV-2) is a new strain of coronavirus causing COVID-19, first identified in Wuhan City, China towards the end of 2019. At present, there is no evidence that pregnant women are more likely to be severely ill, need ICU care, or die from the illness in comparison to non-pregnant adults. Evidences suggest that vertical transmission, might be possible. We searched and retrieved the published literature from PubMed and Google Scholar using various keywords. We further searched the official webpages of various organizations for the updated information.
\end{abstract}

Pregnant individuals in particular are encouraged to take all available precautions to optimize health and avoid exposure to COVID-19. Adequate Testing should be prioritized in pregnant women admitted with suspected COVID-19. When a pregnancy is complicated by critical illness, the pregnant patient should ideally be cared for at a Level III or IV hospital. In the face of COVID-19, antenatal fetal surveillance and ultrasonography should continue as medically indicated when possible and elective ultrasound examinations should not be performed. Maternal immunizations continue to be an essential component of prenatal care and Obgyns should screen all pregnant individuals for mental health issues. Although the most commonly reported sign in COVID-19 is fever, nevertheless, other causes of intrapartum fever should not be overlooked. Cesarean delivery should be based on obstetric indications and not COVID-19 status alone. Infants born to patients with known COVID-19 should be considered infants with suspected COVID-19. The suspected or confirmed COVID-19 mother and infant can be allowed to remain together with enhanced precautions and suspected or confirmed maternal COVID-19 is not a contraindication to breastfeeding. If both the mother and the infant are healthy, it may be prudent to expedite discharge, so as to limit the risk of inadvertent exposure and infection. The Ob-gyns should commit to providing necessary care, although modifications to health care delivery approaches may be necessary.

\section{KEY WORDS}

COVID-19, Maternal health, Pregnancy 


\section{INTRODUCTION}

Novel coronavirus (SARS-CoV-2) is a new strain of coronavirus causing COVID-19, first identified in Wuhan City, China towards the end of 2019. ${ }^{1}$ The global cases of COVID-19 have evidence of human-to-human transmission. The SARS-CoV-2 virus can be readily isolated from respiratory droplets or secretions, faeces and fomites (objects). Transmission of the virus is known to occur most often through close contact with an infected person (within 2 metres) or from contaminated surfaces. ${ }^{2}$ This article revisits the strategies for maternal health care in the face of COVID-19 pandemic.

\section{METHODS}

For preparing this article, we searched and retrieved the published literature from PubMed and Google Scholar using various keywords: "COVID-19/pregnancy/maternal health". We further searched the official webpages of Centres for Disease Control and Prevention (CDC), World Health Organization (WHO), Royal College of Obstetricians and Gynaecologists RCOG), The American College of Obstetricians and Gynaecologists (ACOG), The Royal Australian and New Zealand College of Obstetricians and Gynaecologists, Canadian Paediatric Society, The Society of Obstetricians and Gynaecologists of Canada and other webpages as well as governmental and non-governmental organizations for the updated information.

\section{DISCUSSION}

Transmission: Pregnant women are not more prone to contract COVID-19 than the general population. ${ }^{3-5}$ At present, there is no evidence that pregnant women are more likely to be severely ill, need ICU care, or die from the illness in comparison to non-pregnant adults. ${ }^{6}$

Evidences suggest that vertical transmission (transmission from woman to her baby antenatally or intrapartum), might be possible. ${ }^{7}$ Evidences have been published in a couple of reports regarding immunoglobulin M (IgM) for SARS-CoV-2 in neonatal serum at birth. ${ }^{8,9}$ There has been a recent reporting of a high SARS-CoV-2 viral load in the placenta, which was associated with a viraemia in the mother, subsequently followed by an infection in the neonate, including neurological manifestations with inflammatory changes in the neonatal cerebrospinal fluid. ${ }^{10}$

Evidence from the interim report based on The UK Obstetric Surveillance System (UKOSS), showed that six babies (2.5\%) had a positive nasopharyngeal swab within 12 hours of birth. ${ }^{11}$ A systematic review of 24 pregnant women with COVID-19 was carried out and it revealed that there was no evidence of SARS-CoV-2 on polymerase chain reaction testing of placenta, amniotic fluid, cord blood or breastmilk samples. ${ }^{12}$ A larger systematic review was conducted on 666 neonates born to women with confirmed COVID-19, and it revealed that 28 out of 666 (4\%) neonates had confirmed COVID-19 infection postnatally. In this review, on comparing the mode of delivery, eight out of 292 (2.7\%) neonates were born vaginally and 20 out of 374 (5.3\%) were born via caesarean birth. In this review, seven babies were breast fed, three were formula fed, one was given expressed breast milk and in 17 neonates the method of infant feeding was not reported. The review showed that COVID-19 infection in the neonates is rare and the infection rate is no greater when the baby is born vaginally, breastfed or stays with the mother after birth. ${ }^{13}$ Further investigation around vertical transmission is required and is currently going on.

Effect of COVID-19 on pregnant women: The risk factors for hospital admission with COVID-19 infection in pregnancy include: Black, Asian or minority ethnicity (BAME); Overweight or obesity; Pre-existing comorbidity; Maternal age $>35$ years. ${ }^{14}$ Within the general population, there is increasing evidence that there could be a cohort of asymptomatic individuals or those with very minor symptoms who are carrying the virus, even though the prevalence is unknown. A prospective study of 675 pregnant women admitted consecutively to three New York City hospitals for birth, all tested for SARS-CoV-2, found that $10 \%$ of women were positive for the virus and $79 \%$ of those infected women were asymptomatic for COVID-19. ${ }^{5}$ A majority of the pregnant women will suffer from mild or moderate cold/flu-like symptoms. Cough, fever, shortness of breath, headache and anosmia are other relevant symptoms. ${ }^{15}$ Pneumonia and marked hypoxia are the more severe symptoms commonly described with COVID-19 in older people, the immunosuppressed and those with chronic conditions such as chronic lung disease, cancer or diabetes. ${ }^{16}$ The symptoms of severe infection are similar in pregnant women and early identification and assessment for prompt supportive treatment is key. Another cases series from New York, suggests possible patterns of disease in pregnant women. The case series describes 43 pregnant women who tested positive for SARS-CoV-2 and reported a similar pattern of disease severity to non-pregnant adults: $86 \%$ mild, $9 \%$ severe and $5 \%$ critical, although the sample size was too small to draw a definitive conclusion and no comparison was made for age, sex or co-morbiditymatched individuals. ${ }^{17}$

General Counselling Measures for Pregnant Women: Counselling should be imparted by clinicians to pregnant women and those contemplating pregnancy about the potential risk for severe illness from COVID-19, and emphasis should be laid for pregnant women and their families on measures to prevent infection with SARSCoV-2. Pregnant individuals in particular are encouraged to take all available precautions to optimize health and avoid exposure to COVID-19 including - maintaining prenatal care appointments; wearing a mask and other recommended PPE, if applicable, at work and in public; washing hands frequently; maintaining physical distancing; limiting contact 
with other individuals as much as practicable and maintain an adequate supply of preparedness resources including medications. ${ }^{18}$

Testing: Testing is vital for mitigation of risk, collection of data, and directing critical resources, including PPE. Testing should be prioritized in pregnant women admitted with suspected COVID-19 or those who develop symptoms suggestive of COVID-19 during admission. Health facilities, in addition, may consider additional molecular (eg, PCR by nasopharyngeal swab) testing strategies, such as universal testing as the potential for asymptomatic patients presenting to labor and delivery units exists, especially in areas with high prevalence. ${ }^{18}$

Community mitigation efforts: There has been implementation of community mitigation efforts across countries, to control the spread of COVID-19. These efforts are important, however, ob-gyns should be aware of the unintended effect they may have, including limiting access to routine prenatal care. The Ob-gyns should commit to providing necessary prenatal care, referrals, and consultations, although modifications to health care delivery approaches may be necessary. There can be a possibility of a decreased health care workforce, potential shortage of personal protective equipment, and limited isolation rooms. Therefore ob-gyns should consider creating a plan to address these potential issues, and should maximize the use of telehealth across as many aspects of prenatal care as possible. ${ }^{18}$

Level of Management of Critically III Covid-19 Pregnant Patients: Pregnant patients with comorbidities may be at increased risk for severe illness, similar to the general population with similar comorbidities. The clinical management of COVID-19 pregnant patients includes rapid implementation of the recommended infection prevention and control measures. It also includes the supportive management of complications including critical care. When a pregnancy is complicated by critical illness, the pregnant patient should ideally be cared for at a Level III or IV hospital with obstetric services and an adult ICU. It should be kept in mind that the COVID-19 status alone is not necessarily a reason to transfer non-critically ill pregnant women with suspected or confirmed COVID-19, however, the planning of care location should ideally be based on the levels of maternal and neonatal care. Factors such as lack of adequate staff to care for a critically ill patient, need for special equipment, need for frequent assessments, and the need for access to trials for novel treatments may influence the decision to transfer a patient to a higher level of care. If there is a need to transfer a patient to a higher level of care for facility-level factors, it is prudent that a discussion between the transferring health care practitioner and the intensive care practitioners is held, regarding the current limitations of care on the obstetric unit and such a discussion may help facilitate rapid transfer. ${ }^{19}$
Use of PPE: It is mandatory for all healthcare personnel to wear a facemask at all times while they are in a healthcare facility, in order to protect patients and other health care workers. ${ }^{20}$ As suggested by recent data, healthcare-related COVID-19 infections in the health-care setting can be decreased by universal masking, appropriate use of N95 respirators, and close evaluation of extended use or reuse of $\mathrm{N}-95$ respirators. ${ }^{21,22}$ Healthcare personnel should also wear eye protection in addition to their facemask in areas with moderate to substantial community transmission. ${ }^{23}$ In locations where universal testing is not employed and adequate PPE is available, universal PPE, including respirators such as $\mathrm{N} 95$ respirators is recommended until the patient's status is known. In addition, all medical staff should be trained in proper donning and doffing of PPE and should adhere to it. The data from the SARS outbreak suggest that the comprehensive listing of recommended PPE used along with hand hygiene and environmental cleaning leads to the optimal decreased risk of respiratory virus transmission, and this probably holds true for COVID-19 as well. ${ }^{19}$ In the event that there is a possible N95 shortage, extended use or limited reuse of N95 masks may be implemented or necessary. If extended use or limited reuse is being implemented, polices regarding extended use or limited reuse should be in accordance with Centres for Disease Control (CDC) recommendations, taking into account the actual masks being used. While developing such policies, coordination with local occupational health and infection control departments must be taken into account. ${ }^{19}$ A limited number of research have noted subtle physiologic changes with no known clinical impact associated with extended wear of N95 masks. However, the reduction of infectious risk outweighs any theoretical physiologic concern. ${ }^{24,25}$

Antenatal fetal surveillance and ultrasonography: In the face of COVID-19, antenatal fetal surveillance and ultrasonography should continue as medically indicated when possible. Elective ultrasound examinations should not be performed and ultrasonography should be used wisely and only when its use is expected to answer a relevant clinical question or otherwise provide medical benefit to the patient. ${ }^{26,27}$ If the risk of exposure and infection within the community outweighs the benefit of testing, then it may be appropriate to postpone or cancel some testing or examinations. However, this decision should be made at the local practice or facility level, subsequent to adequately balancing the risks and benefits of decreased exposure, completing the test, and site capacity. Reducing care is only appropriate because the risk of inadvertent exposure from receiving or delivering care can be high at this time and therefore, normal antenatal testing or ultrasonography scheduling should resume when this risk subsides. Any modifications made to care should be conveyed to patients with a discussion of the altered balance of risks and benefits of coming to the facility/hospital for testing 
or ultrasonography in the face of a global pandemic, and should be well documented in the medical record. ${ }^{19}$

Immunization: During the COVID-19 pandemic, maternal immunizations continue to be an essential component of prenatal care. It is encouraged to comply with the recommended timing of maternal immunization as much as possible, so as to maximize maternal and fetal benefits. If a practice decides to modify or reduce the number of prenatal care visits, practitioners are encouraged to include recommended maternal immunizations during remaining in-person appointments, even if that means immunizations will be administered outside of the typically recommended weeks of gestation. ${ }^{28}$

Maternal Mental Health: The most common complications that occur in pregnancy or in the first 12 months after delivery are perinatal mood and anxiety disorders. In the face of COVID-19 pandemic, ob-gyns and other maternal health care professionals should screen all pregnant individuals for symptoms of anxiety and depression with a standardized, validated tool, at least once during the perinatal period. ${ }^{29}$ Even if the woman is screened during pregnancy, additional screening should be carried out during the postpartum period. ${ }^{29}$ Patients should be reassured by ob-gyns and other maternal health care professionals that even during this time, there are effective treatment and support options available. If the patient is at imminent risk of harm to self or others, she must be referred to emergency services for further evaluation.

Intrapartum Fever and COVID-19 Infection: The most commonly reported sign is fever and most patients with confirmed COVID-19 have developed fever and/or symptoms of acute respiratory illness. Nevertheless it must be kept in mind that other causes of intrapartum fever should not be overlooked. Prudent judgement has to be used by clinicians to conclude whether a patient has signs and symptoms compatible with COVID-19 and whether the patient should be tested. There is limited data regarding COVID-19 in pregnancy and as per current information, presenting signs and symptoms are expected to be similar to those for non-pregnant patients, including the presence of fever. ${ }^{19}$ The epidemiologic factors such as the occurrence of local community transmission of COVID-19 infections are other considerations that may guide testing. The clinicians are strictly recommended to test for other causes of respiratory illness and peripartum fever, as part of evaluation. ${ }^{30}$

Timing of Delivery: Maternal COVID-19 infection, in a majority of cases, should not dictate the timing of delivery. No alteration to the usual timing of delivery is indicated for women with suspected or confirmed COVID-19 early in pregnancy who recover. For women with suspected or confirmed COVID-19 in the third trimester who recover, it is reasonable to attempt to postpone delivery (if no other medical indications arise) until a negative testing result is obtained or quarantine status is lifted so as to attempt to avoid transmission to the neonate. In general, COVID-19 infection itself is not an indication for delivery. ${ }^{19}$

Indication for cesarean delivery: Cesarean delivery should be based on obstetric (fetal or maternal) indications and not COVID-19 status alone. As regards considering COVID-19 as an indication for cesarean delivery for patients with suspected or confirmed COVID-19 infection, currently, based on very limited data based on primarily cesarean deliveries, there does not appear to be a risk of vertical transmission via the transplacental route. In addition, based on limited data, outcomes for individuals appear to be similar between pregnant and nonpregnant patients. ${ }^{19}$

Infants born to patients with suspected/confirmed COVID-19: It is to be understood that infants born to patients with known COVID-19 at the time of delivery should be considered infants with suspected COVID-19. Ideally, infants with suspected COVID-19 should be tested, isolated from other healthy infants, and cared for according to the Interim Infection Prevention and Control Recommendations for Patients with Suspected or Confirmed Coronavirus Disease 2019 (COVID-19). ${ }^{31}$ Infants born to a pregnant individual with suspected COVID-19 for whom testing is unknown (either pending results or not tested) are not considered to be infants with suspected COVID-19. Discharge for postpartum individuals with suspected or confirmed COVID-19 should follow recommendations described in CDC's Discontinuation of Transmission-Based Precautions and Disposition of Patients with COVID-19 in Healthcare Settings (Interim Guidance) in conjunction with guidance from the local and state health system. ${ }^{32}$

Location of the Mother-Infant Dyad: It is a challenging issue to decide whether to separate a woman with suspected or confirmed COVID-19 from her newborn because of limited available current evidence on which to base firm recommendations. Several organizations have recommended allowing the mother and infant to remain together with enhanced precautions in place when the mother has suspected or confirmed COVID-19. This reinforces the well-documented benefits of keeping the mother with her newborn, breastfeeding, and skin-toskin contact. ${ }^{33-36}$ Considering the fact that there is limited evidence on this topic, the determination of whether to keep patients with known or suspected COVID-19 and their infants together or to separate after birth should be made on a case-by-case basis. This should be done by using shared decision-making between the patient and the clinical team. The counselling process should include a discussion of the risks and benefits, including the benefits of keeping the mother with the newborn and the risk of uncommon but potentially severe infection. Separation in the hospital setting temporarily reduces the risk of neonatal infection. However, separation of patients from their newborns may be linked to additional risks which include disruption of breastfeeding and undue stress on the patient. Additionally, discussions should be held on available infection control 
measures and options for accommodations, including feasibility of temporary separation in both the hospital setting and upon discharge. ${ }^{18}$

Breastfeeding: Breastmilk provides protection against many illnesses and there are few contraindications to breastfeeding. ${ }^{37,38}$ It is not known whether COVID-19 can be transmitted through breastmilk, or if any potential viral components, if transmitted, are infectious. A recent case report has detected SARS-CoV-2 RNA in the breastmilk, however, most of the data has not demonstrated the presence of SARS-CoV-2 virus in breastmilk. ${ }^{39}$ Therefore, suspected or confirmed maternal COVID-19 is not considered a contraindication to infant feeding with breastmilk at this time. It should be kept in mind that women with suspected or confirmed COVID-19 can transmit the virus through respiratory droplets while in close contact with the infant, including while breastfeeding. Therefore, it is mandatory for obstetrician-gynecologists to counsel women with suspected or confirmed COVID-19 who intend to breastfeed, on how to minimize the risk of transmission.

\section{Considering expedited discharge during the COVID-19} pandemic: If both the mother and the infant are healthy, it may be prudent to expedite discharge, so as to limit the risk of inadvertent exposure and infection. ${ }^{40}$ The discharge may be considered after 1 day for women with uncomplicated vaginal births and after 2 days for women with cesarean births depending on their status. Early discharge will necessitate discussion with the facility's pediatric care team and should be linked to home telehealth visits for the mother and infant. ${ }^{19}$ In addition, it is vital to offer mental health services, especially for patients who are experiencing anxiety regarding the COVID-19 pandemic or are at an increased risk of intimate partner violence.

\section{REFERENCES}

1. World Health Organisation. Coronavirus disease (COVID-2019) situation reports 2020. Available at: https://www.who.int/ emergencies/diseases/novel-coronavirus-2019/situation-reports / accessed - August 9, 2020.

2. World Health Organisation. Coronavirus disease (COVID-2019) situation reports 2020 Available at: https://www.who.int/newsroom/commentaries/detail/transmission-of-sars-cov-2-implicationsfor-infection-prevention-precautions / accessed - August 9, 2020.

3. Docherty $A B$, Harrison $E M$, Green $C A$, et al. Features of 16,749 hospitalised UK patients with COVID-19 using the ISARIC WHO Clinical Characterisation Protocol. medRxiv. 2020:2020.04.23.20076042. doi:10.1101/2020.04.23.20076042

4. Smith $V$, Seo $D$, Warty $R$, et al. Maternal and neonatal outcomes associated with COVID-19 infection: A systematic review. PLoS One. 2020;15(6):e0234187. doi: 10.1371/journal.pone.0234187. eCollection 2020

5. Prabhu M, Cagino K, Matthews KC, et al. Pregnancy and postpartum outcomes in a universally tested population for SARS-CoV-2 in New York City: A prospective cohort study. BJOG. 2020; 10.1111/14710528.16403. doi: 10.1111/1471-0528.16403.

6. ICNARC. Report on COVID-19 in critical care 2020. Available at: https://www.icnarc.org/Our-Audit/Latest-News/2020/04/10/ReportOn-5578-Patients-Critically-III-With-Covid-19, accessed August 9, 2020.
Modifying post-partum care delivery approaches to decrease the risk of COVID-19 exposure: It is considered appropriate to modify postpartum care delivery approaches to decrease the risk of COVID-19 exposure. However, it has to be kept in mind that modifying or reducing care is only appropriate because the risk of inadvertent exposure from receiving or delivering care can be high at this time. Therefore, normal care approaches and schedules should resume when this risk subsides. Plans for modified care are best made at the local level with consideration of patient populations and available resources. Some examples of approaches to modifying postpartum care that may be considered are - Perform the initial three week (or sooner) assessment, wound checks, and blood pressure checks by phone or telehealth visits, if possible; delay the comprehensive postpartum visit to 12 weeks, with the intention of seeing the patient for the comprehensive assessment in person and using telehealth visits as needed before 12 weeks. ${ }^{19}$

\section{CONCLUSION}

The Ob-gyns should commit to providing necessary prenatal care, referrals, and consultations, although modifications to health care delivery approaches may be necessary. There can be a possibility of a decreased health care workforce, potential shortage of personal protective equipment, and limited isolation rooms. Therefore ob-gyns should consider creating a plan to address these potential issues, and should maximize the use of telehealth across as many aspects of prenatal care as possible.

7. Lamouroux A, Attie-Bitach T, Martinovic J, et al. Evidence for and against vertical transmission for SARS-CoV-2 (COVID-19). Am J Obstet Gynecol. 2020; 223(1):91.e1-91.e4. doi: 10.1016/j.ajog.2020.04.039.

8. Dong L, Tian J, He S, et al. Possible Vertical Transmission of SARSCoV-2 From an Infected Mother to Her Newborn. JAMA. 2020 Mar 26;323(18):1846-1848.doi: 10.1001/jama.2020.4621.

9. Zeng $\mathrm{H}, \mathrm{Xu} \mathrm{C}$, Fan J, et al. Antibodies in Infants Born to Mothers With COVID-19 Pneumonia. JAMA. 2020;323(18):1848-1849. doi: 10.1001/ jama.2020.4861.

10. Vivanti AJ, Vauloup-Fellous C, Prevot S, et al. Transplacental transmission of SARS-CoV-2 infection. Nat Commun. 2020;11(1):3572. doi: $10.1038 / s 41467-020-17436-6$

11. Knight M, Bunch K, Vousden N, et al. Characteristics and outcomes of pregnant women admitted to hospital with confirmed SARSCoV-2 infection in UK: national population based cohort study. BMJ. 2020;369:m2107. doi: 10.1136/bmj.m2107

12. Martins-Filho PR, Santos VS, Santos HP, Jr. To breastfeed or not to breastfeed? Lack of evidence on the presence of SARS-CoV-2 in breastmilk of pregnant women with COVID-19. Rev Panam Salud Publica. 2020;44:e59. doi: 10.26633/RPSP.2020.59

13. Walker KF, O'Donoghue K, Grace N, et al. Maternal transmission of SARS-COV-2 to the neonate, and possible routes for such transmission: A systematic review and critical analysis. BJOG. 2020;10.1111/14710528.16362.doi: 10.1111/1471-0528.16362. 
14. Coronavirus (COVID-19) Infection in Pregnancy. Information for healthcare professionals, Version 11: Royal College of Obstetricians \& Gynaecologists; 2020. Available at: https://www.rcog.org.uk/ globalassets/documents/guidelines/2020-07-24-coronavirus-covid19-infection-in-pregnancy.pdf / accessed - August 9, 2020

15. Public Health England. COVID-19: investigation and initial clinical management of possible cases 2020. Available at: https://www. gov.uk/government/publications/wuhan-novel-coronavirus-initialinvestigation-of-possible-cases/investigation-and-initial-clinicalmanagement-of-possible-cases-of-wuhan-novel-coronavirus-wn-covinfection/accessed August 9, 2020

16. Guan W-j, Ni Z-y, Hu Y, et al. Clinical Characteristics of Coronavirus Disease 2019 in China. N Engl J Med. 2020;382(18):1708-1720. doi: 10.1056/NEJMoa2002032.

17. Breslin N, Baptiste C, Gyamfi-Bannerman C, et al. COVID-19 infection among asymptomatic and symptomatic pregnant women: Two weeks of confirmed presentations to an affiliated pair of New York City hospitals. Am J Obstet Gynecol MFM. 2020;2(2):100118.doi: 10.1016/j.ajogmf.2020.100118.

18. General Information Regarding Pregnant Individuals and COVID-19. The American College of Obstetricians and Gynecologists. Available at : https://www.acog.org/clinical/clinical-guidance/practice-advisory/ articles/2020/03/novel-coronavirus-2019 / accessed - August 9, 2020

19. COVID-19 FAQs for Obstetrician Gynecologists, Obstetrics. The American College of Obstetricians and Gynecologists. Available at: https://www.acog.org/clinical-information/physician-faqs/covid-19faqs-for-ob-gyns-obstetrics / accessed-August 9, 2020

20. Clinical Questions about COVID-19: Questions and Answers. Centres for Disease Control and Prevention. Available at: https:// www.cdc.gov/coronavirus/2019-ncov/hcp/faq.html?CDC_AA_ refVal=https\%3A\%2F\%2Fwww.cdc.gov\%2Fcoronavirus\%2F $2019-$ ncov\%2Fhcp\%2Finfection-control-faq.html / accessed - August 9, 2020

21. Degesys NF, Wang RC, Kwan E, et al. Correlation Between N95 Extended Use and Reuse and Fit Failure in an Emergency Department. JAMA. 2020;324(1):94-96. doi:10.1001/jama.2020.9843

22. Chu DK, Akl EA, Duda S, et al. Physical distancing, face masks, and eye protection to prevent person-to-person transmission of SARSCoV-2 and COVID-19: a systematic review and meta-analysis. Lancet. 2020;395(10242):1973-1987. doi: 10.1016/S0140-6736(20)31142-9

23. Interim Infection Prevention and Control Recommendations for Healthcare Personnel During the Coronavirus Disease 2019 (COVID-19) Pandemic. Centres for Disease Control and Prevention. Available at: https://www.cdc.gov/coronavirus/2019-ncov/hcp/ infection-control-recommendations.html / accessed - August 9, 2020

24. Kim, J., Roberge, R.J. \& Powell, J.B. Effect of external airflow resistive load on postural and exercise-associated cardiovascular and pulmonary responses in pregnancy: a case control study. BMC Pregnancy Childbirth. 2015;15:45.

25. Tong PSY, Kale AS, Ng K et al. Respiratory consequences of N95-type Mask usage in pregnant healthcare workers-a controlled clinical study. Antimicrob Resist Infect Control. 2015;4:48. doi: 10.1186/ s13756-015-0086-z. eCollection 2015.

26. Ultrasound in Pregnancy. Practice Bulletin. Number 175. The American College of Obstetricians and Gynaecologists. Available at: https://www.acog.org/clinical/clinical-guidance/practice-bulletin/ articles/2016/12/ultrasound-in-pregnancy / accessed - August 9, 2020

27. Guidelines for Diagnostic Imaging During Pregnancy and Lactation. Committee Opinion. Number 723. The American College of Obstetricians and Gynaecologists. Available at: https://www.acog. org/clinical-information/physician-faqs/ /link.aspx?_id=7310B1BE69 B84FB9B5463D32BBC7BBF9\&_z=z / accessed - August 9, 2020
28. Maternal Immunization. Committee Opinion. Number 741. The American College of Obstetricians and Gynaecologists. Available at: https://www.acog.org/clinical-information/physician-faqs/ /link. aspx?_id=E996987F2E81491F8009AFA85D2DC1D3\&_z=z / accessed - August 9, 2020

29. Screening for Perinatal Depression. Committee Opinion. Number 757. The American College of Obstetricians and Gynaecologists. Available at: https://www.acog.org/clinical-information/physician-faqs/ /link. aspx?_id=F98D690269D840209E0D82B454EE7463\&_z=z / accessed - August 9, 2020

30. Overview of Testing for SARS-CoV-2. Centres for Disease Control and Prevention. Available at: https://www.cdc.gov/ coronavirus/2019-ncov/hcp/testing-overview.html?CDC_AA_ refVal=https\%3A\%2F\%2Fwww.cdc.gov\%2Fcoronavirus\%2F2019ncov\%2Fhcp\%2Fclinical-criteria.html/accessed - August 10, 2020

31. Interim Infection Prevention and Control Recommendations for Healthcare Personnel During the Coronavirus Disease 2019 (COVID-19) Pandemic. Centres for Disease Control and Prevention. Available at: https://www.cdc.gov/coronavirus/2019-ncov/hcp/ infection-control-recommendations.html/accessed - August 10, 2020

32. Discontinuation of Transmission-Based Precautions and Disposition of Patients with COVID-19 in Healthcare Settings (Interim Guidance). Centres for Disease Control and Prevention. Available at: https:// www.cdc.gov/coronavirus/2019-ncov/hcp/disposition-hospitalizedpatients.html/accessed - August 10, 2020

33. COVID-19 and Breastfeeding. Position Paper. World Health Organization. Available at: https://www.euro.who.int/_data/assets/ pdf_file/0010/437788/breastfeeding-COVID-19.pdf?ua=1 / accessed - August 10, 2020

34. COVID-19 in Pregnancy. Committee Opinion No. 400.The Society of Obstetricians and Gynaecologists of Canada. Available at: https:// www.sogc.org/en/content/featured-news/Committee-Opinion-No400\%E2\%80\%93COVID-19-in-Pregnancy-updated-May-14-2020.aspx / accessed - August 10, 2020

35. Breastfeeding when mothers have suspected or proven COVID-19. Canadian Paediatric Society. Available at: https://www.cps.ca/en/ documents/position/breastfeeding-when-mothers-have-suspectedor-proven-covid-19 / accessed - August 10, 2020

36. A message for pregnant women and their families. The Royal Australian and New Zealand College of Obstetricians and Gynaecologists. Available at: https://ranzcog.edu.au/statements-guidelines/covid-19statement/information-for-pregnant-women / accessed-August 10, 2020

37. Optimizing Support for Breastfeeding as Part of Obstetric Practice. Committee Opinion. Number 756. The American College of Obstetricians and Gynaecologists. Available at: https://www.acog. org/clinical/clinical-guidance/committee-opinion/articles/2018/10/ optimizing-support-for-breastfeeding-as-part-of-obstetric-practice / accessed - August 10, 2020

38. If You Are Pregnant, Breastfeeding, or Caring for Young Children. Centres for Disease Control and Prevention. Available at: https://www. cdc.gov/coronavirus/2019-ncov/need-extra-precautions/pregnancybreastfeeding.html?CDC_AA_refVal=https\%3A\%2F\%2Fwww.cdc. gov\%2Fcoronavirus\%2F2019-ncov\%2Fprepare\%2Fpregnancybreastfeeding.html/accessed - August 10, 2020

39. Groß R, Conzelmann C, Müller JA, Stenger S, Steinhart K, Kirchhoff F, Münch J. et al. Detection of SARS-CoV-2 in human breastmilk. Lancet. 2020;395(10239):1757-1758. doi: 10.1016/S0140-6736(20)31181-8. Epub 2020 May 21.

40. Hospital Disaster Preparedness for Obstetricians and Facilities Providing Maternity Care. The American College of Obstetricians and Gynaecologists. Available at: https://www.acog.org/clinicalinformation/physician-faqs/ /link.aspx?_id=6E897D0CA3144DB1A9 B7EB5FC1B5DC46\&_z=z / accessed - August 10, 2020 